\title{
MTA2 enhances colony formation and tumor growth of gastric cancer cells through IL-11
}

Chenfei Zhou ${ }^{2 \dagger}$, Jun Ji ${ }^{2 \dagger}$, Qu Cai ${ }^{2}$, Min Shi ${ }^{2}$, Xuehua Chen ${ }^{2}$, Yingyan Yu ${ }^{2}$, Zhenggang Zhu ${ }^{1,2}$ and Jun Zhang ${ }^{1 *}$

\begin{abstract}
Background: We have preliminarily reported MTA2 expression in gastric cancer and its biological functions by using knockdown cell models, while the molecular mechanisms of MTA2 in regulating malignant behaviors are still unclear.

Methods: MTA2 overexpression models were established by transfection assay in gastric cancer cells BGC-823 and MKN28. Cell proliferation assay, colony formation in soft agar, wound-healing assay and transwell migration assay were performed with MTA2 overexpression and negative control (NC) cells. Subcutaneous xenografts and pulmonary metastasis models by BGC-823/MTA2 and BGC-823/NC cells were used to observe the capacity of growth and metastasis in vivo. Differential gene expression in MTA2 knockdown and overexpression cells was analyzed by microarrays. IL-11, which demonstrated as differential expression in microarray, was detected by real-time PCR, western blot, ELISA and immunohistochemistry staining. Recombinant human IL-11 (rhIL-11) was administrated in cell proliferation and colony formation as rescue assay.

Results: The numbers of colonies in soft agar were significantly more in BGC-823/MTA2 and MKN28/MTA2 cells, comparing with those in their NC cells. Capabilities of cell proliferation, wound-healing and cell migration were not significantly changed in MTA2 overexpression cells. The sizes of subcutaneous xenografts and pulmonary metastases of BGC-832/MTA2 cells were significantly larger than those in BGC-823/NC group. Differential expression of IL-11 was identified by genome expression microarray both in MTA2 knockdown and overexpression cells. IL-11 expression was elevated in BGC-823/MTA2 cells, whereas reduced in SGC-7901/shMTA2 cells. Administration of rhlL-11 recovered colony formation capacity of SGC-7901/shMTA2 cells.

Conclusions: MTA2 overexpression enhances colony formation and tumor growth of gastric cancer cells, but not plays important role in cancer cell migration and metastasis. IL-11 is one of the downstream effectors of MTA2 in regulating gastric cancer cells growth.
\end{abstract}

Keywords: Gastric cancer, MTA2, Colony formation, Tumor growth, IL-11

\section{Background}

Gastric cancer is one of the most malignant gastrointestinal neoplasms in the world [1]. In the past decades, although its incidence is decreasing worldwide, gastric cancer is still one of the leading causes of cancer-specific mortality in mainland of China [2]. To improve the outcome of gastric cancer patients, mechanisms of tumorigenesis and progression are under investigation, and intent to clinical translation. However, due to the multiple

\footnotetext{
*Correspondence: jun_zj10977@163.com

'Equal contributors

'Department of Oncology, Ruijin Hospital, Shanghai Jiaotong University

School of Medicine, No. 197 Ruijin er Road, Shanghai 200025, China
}

Full list of author information is available at the end of the article molecular aberrances and high heterogeneity, relevant molecular events of gastric cancer cells are far from elucidated [3].

Metastasis associated 1 family, member 2 (MTA2) gene is classified to metastasis associated gene family [4]. Like MTA1, aberrant expression of MTA2 has been reported in several tumors, including ovarian, lung and pancreatic cancer [5-7]. We have previously reported that MTA2 gene was overexpressed in gastric cancer tissues, correlating with tumor invasion, lymph node metastasis, and advanced TNM stage. MTA2 knockdown significantly inhibited gastric cancer cell invasion and metastasis [8]. Yet, its molecular mechanisms are still unclear.

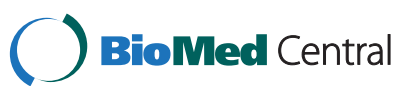

(c) 2015 Zhou et al.; licensee BioMed Central. This is an Open Access article distributed under the terms of the Creative Commons Attribution License (http://creativecommons.org/licenses/by/4.0), which permits unrestricted use, distribution, and reproduction in any medium, provided the original work is properly credited. The Creative Commons Public Domain Dedication waiver (http://creativecommons.org/publicdomain/zero/1.0/) applies to the data made available in this article, unless otherwise stated. 
As a component of the nucleosome remodeling and histone deacetylation (NuRD) complex, MTA2 is necessary for assembly of a catalytically active histone deacetylase (HDAC) complex. HDAC activity is significantly impaired without MTA2 [9]. Nucleosome remodeling and histone deacetylation are the major processes for gene transcriptional regulation [10]. Furthermore, MTA2 has been reported to form transcription complexes with some transcription factors, such as ER $\alpha$ and Twist [11]. Those results indicate that impacts of MTA2 on biological function might via regulating specific genes which were related with tumor malignant behaviors.

In present study, to validated MTA2 biological function, MTA2 overexpression cell models established by MTA2 low expression gastric cancer cells were investigated. Differential gene expression in MTA2 overexpression and knockdown cells was analyzed to identify its downstream effectors. Meanwhile, the biological functions of MTA2-downstream effectors were reversely confirmed through rescue assay.

\section{Methods}

\section{Cell lines and reagents}

Gastric cancer cell lines BGC-823 and MKN28 were preserved in Shanghai Institution of Digestive Surgery. MTA2 knockdown cell SGC-7901/shMTA2 and its negative control cell SGC-7901/NC were described in previous study [8]. All cells were cultured at $37^{\circ} \mathrm{C}$ with $5 \% \mathrm{CO}_{2}$ in RPMI-1640 medium with $10 \%$ fetal calf serum. Selection antibiotics, puromycin or hygromycin, were added into medium if necessary.

Puromycin (Sigma) and hygromycin (Sigma) were respectively dissolved by phosphate buffered saline (PBS) at concentrations of $1 \mathrm{mg} / \mathrm{ml}$ and $200 \mathrm{mg} / \mathrm{ml}$. Recombinant human interleukin-11 (rhIL-11, R\&D systems) was reconstituted following user manual at a concentration of $50 \mu \mathrm{g} / \mathrm{ml}$. SAHA (Selleckchem) was dissolved by DMSO at a concentration of $200 \mathrm{mM}$. All reagents were divided into aliquots, and were stored at $-80^{\circ} \mathrm{C}$.

\section{Establishment of MTA2 overexpression cell models}

Full-length cDNA sequence of MTA2 gene (Gene ID: 9219) was cloned and constructed into vector with Flagtag. MTA2 plasmid and its negative control were packaged into lentivirus, and were used to infect BGC-823 and MKN28 cells, respectively. Stable transfected cells were selected by medium with hygromycin $(200 \mu \mathrm{g} / \mathrm{ml})$. MTA2 overexpression cell models were named as BGC823/MTA2 and MKN28/MTA2, and negative controls were named as BGC-823/NC and MKN28/NC.

\section{RNA extraction and real-time PCR}

Total cell RNA was extracted by Trizol reagent, and their concentrations were determined by microplate spectrophotometer (BioTek). Reverse transcription was performed following protocol of Applied Biosystems. Real-time PCR was performed by ABI Prism 7900HT sequence detection system (Applied Biosystems). Primers for real-time PCR were MTA2: F-TGT ACC GGG TGG GAG ATT AC, R-TGA GGC TAC TAG AAA TGT CCC TG; IL-11: F- ACA GCT GAG GGA CAA ATT CC, R- AGC ACA CCT GGG AGC TGT AG. GAPDH was used as reference gene.

\section{Western blot}

Whole cell proteins were extracted by RIPA reagent (Solarbio), and protein concentrations were determined by DC protein assay (Bio-Rad). Denatured samples were fractionated by SDS-PAGE gel and transferred to PVDF membranes. Membranes were blocked by skim milk for $1 \mathrm{~h}$ at room temperature. Primary antibodies, MTA2 (1:1000, BETHYL), IL-11 (1:200, Santa cruz), $\beta$-actin (1:5000, Sigma) and GAPDH (1:5000, KANGCHEN), were incubated overnight at $4{ }^{\circ} \mathrm{C}$. Fluorescent secondary antibodies $(1: 15000$, LI$\mathrm{COR}$ ) were incubated at room temperature for $1 \mathrm{~h}$. Protein bands were visualized by infrared imaging system (LI-COR).

\section{Immunofluorescence}

Attached cells were fixed by $4 \%$ paraformaldehyde for $30 \mathrm{~min}$, and penetrated by Triton X-100 for $15 \mathrm{~min}$, then blocked by BSA for 30 min. DYKDDDK Tag (9A3) mouse $\mathrm{mAb}$ (1: 100, Cell signaling) was incubated at room temperature for $1 \mathrm{~h}$. After rinsed by PBS, Anti-mouse IgG1 FITC (1:100, eBioscience) was incubated for $30 \mathrm{~min}$. Slides were mounted by VECTASHIELD Mounting Medium with DAPI (Vector Labs). Images were captured by confocal microscope, with Zeiss LSM Image Examiner (Carl Zeiss).

\section{Cell proliferation assay}

One thousand cells per well were plated into 96-well plates, and were cultured overnight. CCK-8 (Dojindo) were added and incubated at $37^{\circ} \mathrm{C}$ for $2 \mathrm{~h}$. Optical density value was detected by microplate spectrophotometer (BioTek). Cell growth curves were drawn.

\section{Soft agar colony formation assay}

Two milliliter medium with $0.4 \%$ agar was used as bottom gel. One thousand cells were suspended in $4 \mathrm{ml}$ medium with $0.2 \%$ agar, and were poured on bottom gel. After 2 weeks, colonies were stained by MTT. Number of colonies was counted at low power lens of microscopy. Size of colonies was analyzed by Colony Counter software (Tanon). RhIL-11 was added at a concentration of $100 \mathrm{ng} / \mathrm{ml}$.

\section{Wound healing assay and migration assay}

Cells were cultured as monolayer in 12-well plates. On reaching more than 95\% confluent, wounds were scratched by pipette tips. After washed by PBS, wounds 
were photographed every $24 \mathrm{~h}$. Cells were cultured by serum-free medium during the process.

Fifty thousand cells suspended in $100 \mu \mathrm{l}$ serum-free medium were added into upper chambers of Transwell (8 $\mu \mathrm{m}$ for 24-well plate, Millipore), and full medium were added into lower chambers. After $24 \mathrm{~h}$, cells were fixed by formalin, and stained by $0.1 \%$ crystal violet.

\section{Nude mouse xenografts}

BGC-823/MTA2 and BGC-823/NC cells were studied in vivo. All mouse experiments were approved by the Animal Care and Use Committee and conducted an accord with the Guide for the Care and Use Laboratory Animals of Ruijin Hospital, Shanghai Jiaotong University School of Medicine. Eight 4-week-old male Balb/c nude mice (Institute of Zoology, Chinese Academy of Sciences) were randomly divided into two groups before establishment of subcutaneous xenografts. One million cells in $100 \mu \mathrm{l}$ PBS were injected into subcutaneous tissues of right side back. After tumor length exceeded $4 \mathrm{~mm}$, tumors were measured every 7 days for 4 weeks, and tumor volume was calculated by following formula: $\mathrm{V}=\left(\right.$ Width ${ }^{2} \times$ Length $) / 2$. At $5^{\text {th }}$ week, tumor nodules were collected, and fixed by $10 \%$ formalin.

In experimental metastasis assay, twenty Balb/c nude mice (Institute of Zoology, Chinese Academy of Sciences) were randomly divided into two groups. One million BGC823/MTA2 or BGC-823/NC cells in $100 \mu \mathrm{l}$ PBS were injected through tail vein, respectively. One mouse of each group was sacrificed after one month to assess metastasis formation. After two months, all mice were dissected. Pulmones were fixed by $10 \%$ formalin. Metastatic lesions were counted in gross specimens, and were confirmed by HE staining.

\section{Immunohistochemistry staining (IHC)}

Fixed tumor nodules were embedded by paraffin, and were made into $4 \mu \mathrm{m}$-thick slices. IHC were performed following EnVision two-step procedure of Dako ${ }^{\mathrm{Ts}} \mathrm{REAL}^{\mathrm{Tm}}$ Envision Detection System (Dako). Primary antibodies included MTA2 (1:200, Santa Cruz), Ki-67 (1:50, Dako), IL-11 (1:100, Santa Cruz). Slices were visualized by diaminobenzidine.

\section{Microarray data}

Differential gene expression between BGC-823/NC and BGC-823/MTA2, SGC-7901/NC and SGC-7901/shMTA2 cells was analyzed, respectively. Total RNA of cells were extracted by Trizol reagent. Whole human genome microarray $4 \times 44 \mathrm{~K}$ (Design ID: 014850, Agilent technologies) was used to detect gene expression profile following manufacturer's instructions. Raw data were normalized by Quantile algorithm using Gene Spring Software 11.0 (Agilent technologies). Genes with fold change $>2$ or $<0.05$ and $\mathrm{p}$-value $<0.05$ were identified. Microarray data are available in the ArrayExpress database (www.ebi.ac.uk/ arrayexpress) under accession number E-MTAB-3469.

\section{Enzyme-linked immunosorbent assay (ELISA)}

Supernatants of SGC-7901/NC and SGC-7901/shMTA2 cells, BGC-823/NC and BGC-823/MTA2 cells were collected, and were centrifuged to remove cell debris. Total levels of IL-11 protein were measured by using human IL-11 antibody (R\&D system, MAB618). IL-11 levels were normalized to amount of mRNA in each sample.

\section{Statistics}

Independent-samples $t$ test, Mann-Whitney $\mathrm{U}$ test and one-way ANOVA test (LSD post-hoc) were used to analyze quantitive data. $P$-value $<0.05$ was considered as statistical significant $\left(*: \mathrm{P}<0.05 ;{ }^{* *}: \mathrm{P}<0.01\right)$. The error bars in all figures represented standard deviation. All tests were preformed by SPSS 13.0 software (SPSS Inc.).

\section{Results}

MTA2 overexpression promoted cell colony formation in soft agar

Introduction of MTA2 into BGC-823 and MKN28 cells was confirmed by both real-time PCR and western blot (Figure 1A). Immunofluorescence staining demonstrated that exogenous MTA2 protein was localized in cell nucleus (Figure 1B).

Growth curves of BGC-823/MTA2 and MKN28/MTA2 cells were similar, as compared with those in NC cells (Figure 2A). In soft agar colony formation assay, more colonies were observed in MTA2 overexpression cells (MTA2 vs. NC, BGC823: $15.8 \pm 2.4$ vs. $9.0 \pm 1.2, P=0.002$; MKN28: $21.0 \pm 1.8$ vs. $14.3 \pm 1.0, P=0.001$, Figure $2 B$ ).

No differences were found between BGC-823/MTA2 and BGC-823/NC cells in wound-healing courses, as well as those in MKN28/MTA2 and its NC cells (Figure $2 \mathrm{C}$ ). In migration assay, the numbers of cells passed through membrane were also similar between MTA2 overexpression and NC cells (MTA2 vs. NC, BGC-823: $17.0 \pm 2.6$ vs. $15.7 \pm 2.5, P>0.05$; MKN28: $3.0 \pm 1.0$ vs. $4.0 \pm 1.0, P>0.05$, Figure 2D).

\section{MTA2 overexpression enhanced xenografts and pulmonary metastases growth}

Growth rate of subcutaneous xenografts in BGC-823/ MTA2 group was significantly faster than that in BGC823/NC group. At fourth week, tumor volume and weight were significantly larger in BGC-823/MTA2 group than those in NC group (volume: 2885.7 \pm $1109.8 \mathrm{~mm}^{3}$ vs. $1509.6 \pm 133.2 \mathrm{~mm}^{3}, P=0.021$; weight: $1.51 \pm 0.58$ g vs. $0.86 \pm 0.17, P=0.038$, Figure $3 \mathrm{~A}$ ). Ki-67 staining was stronger in BGC-823/MTA2 xenografts than that in $\mathrm{NC}$ (Figure 3B). 


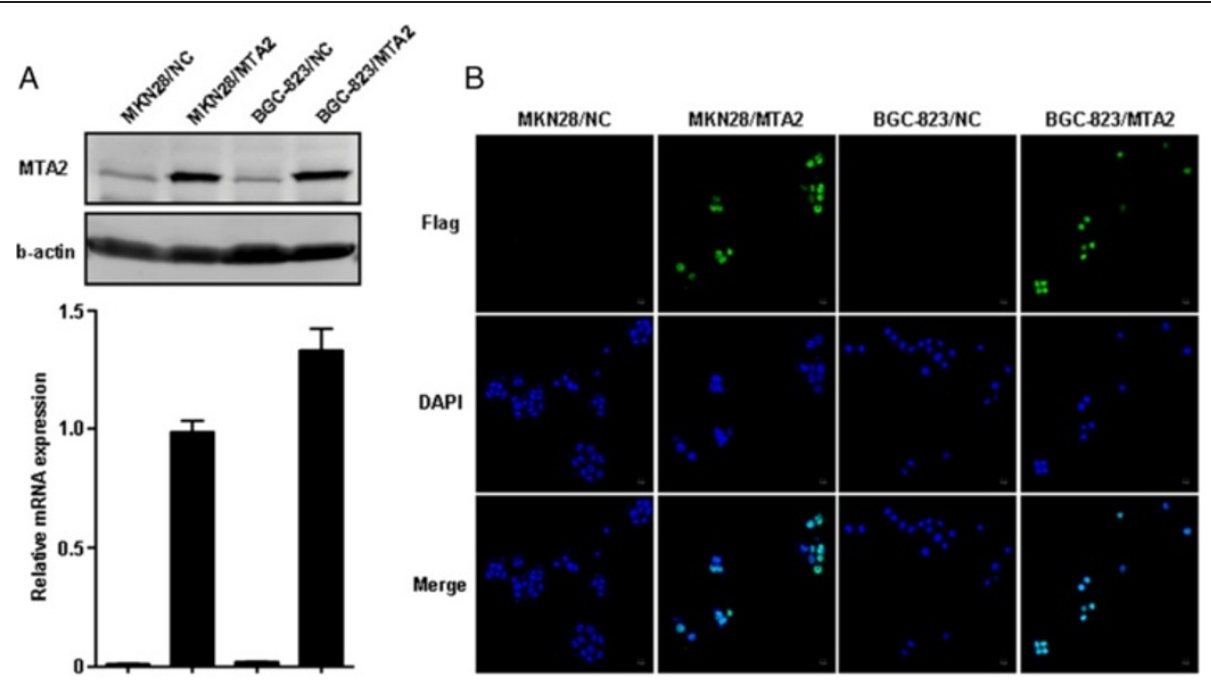

Figure 1 Establishment of MTA2 overexpression gastric cancer cell models. A: MTA2 expression in BGC-823 and MKN28 cells was detected by real-time PCR and western blot. B: Exogenous MTA2 protein was localized in nucleus (immunofluorescence). "Immunofluorescence staining demonstrated that exogenous MTA2 protein was localized in cell nucleus (Figure 1B)." "High magnification image was provided in Additional file 1: Figure S1."

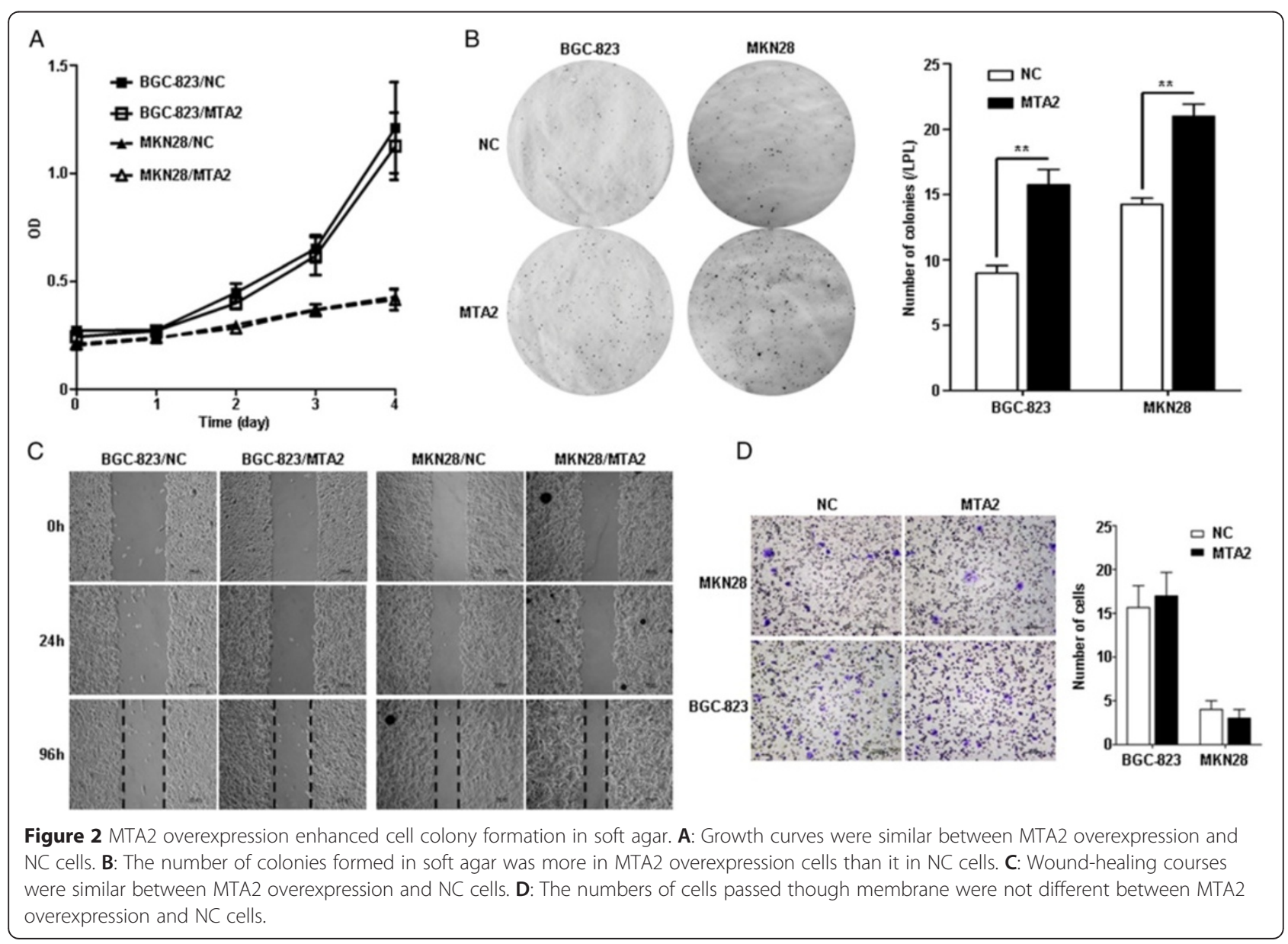




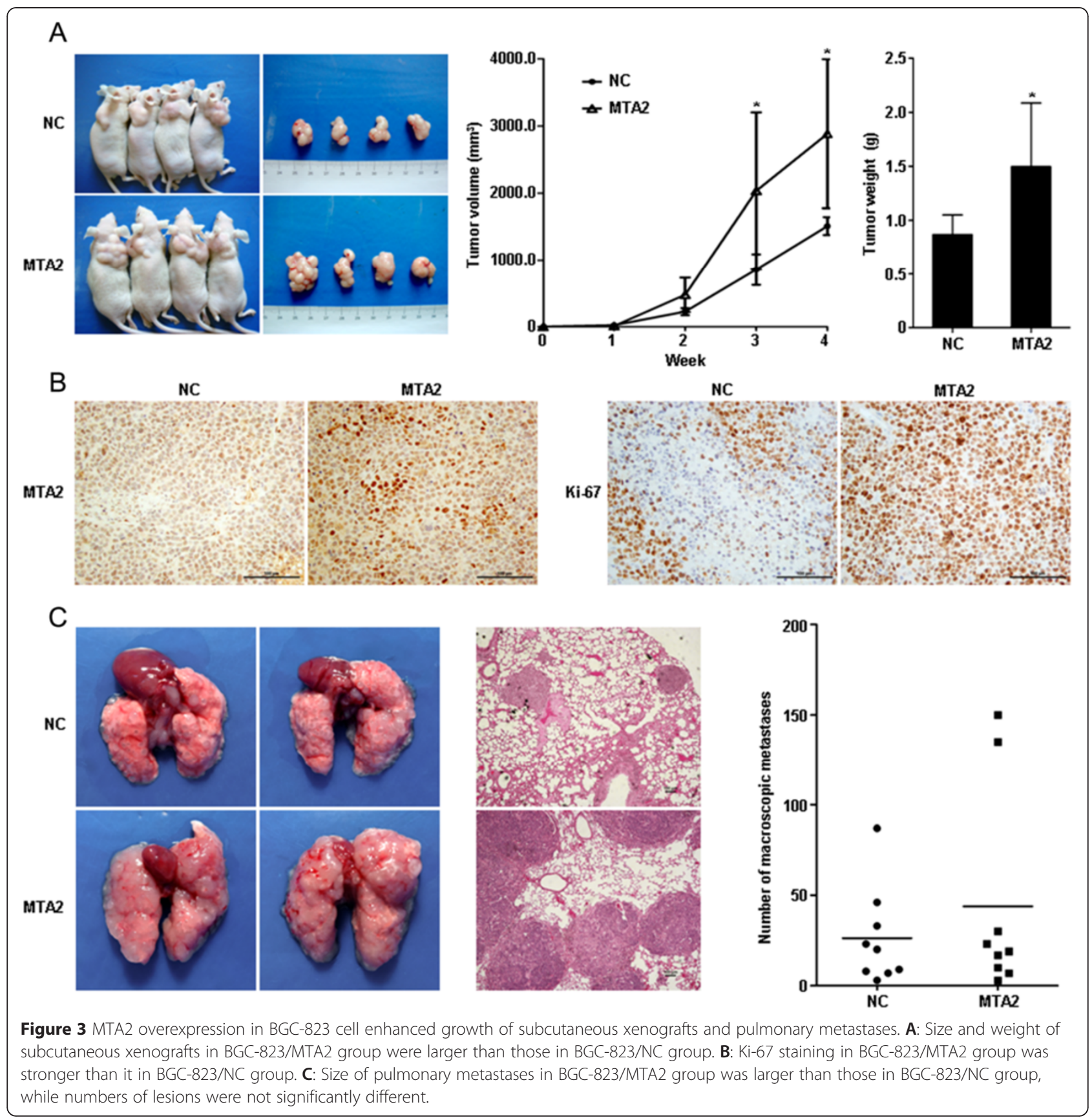

Pulmonary metastases were observed in all mice. In BGC-823/MTA2 group, the volume of metastatic lesions was larger than those in BGC-823/NC group (Figure 3C). However, the number of metastases was no difference between those two groups (MTA2 vs. NC: $43.8 \pm 56.7$ vs. $26.2 \pm 26.7, P>0.05$, Figure 3 C).

\section{IL-11 was identified as a differential expressed gene by} microarray

Differential gene expression between BGC-823/MTA2 and BGC-823/NC cells as well as SGC-7901/shMTA2 and SGC-7901/NC cells were analyzed. Overlapped differential genes between those two data sets were identified. IL-11 demonstrated the most significant fold changes in both data sets (Table 1).

IL-11 mRNA was significantly changed in SGC-7901/ shMTA2 and BGC-823/MTA2 cells, as the results of microarray $(\mathrm{P}<0.01$, Figure $4 \mathrm{~A})$. IL-11 protein was also reduced in SGC-7901/shMTA2 cells, and slightly elevated in BGC-823/MTA2 cells by western blot, as well as in ELISA test (SGC-7901/NC vs. SGC-7901/shMTA2: $1.89 \pm 0.33$ vs. $0.81 \pm 0.35, \mathrm{P}<0.01 ; \mathrm{BGC}-823 / \mathrm{NC}$ vs. 
Table 1 Differential gene expression in MTA2 overexpression and knockdown cells

\begin{tabular}{|c|c|c|c|c|c|}
\hline \multirow[t]{2}{*}{ Gene } & \multicolumn{2}{|c|}{ MTA2 overexpression } & \multicolumn{2}{|c|}{ MTA2 knockdown } & \multirow[t]{2}{*}{ Description } \\
\hline & $\mathrm{Fc}$ & $P$ value & Fc & $P$ value & \\
\hline $\mid \mathrm{L}-11$ & 7.3145 & 7.74E-06 & 0.2631 & 0.0054 & Interleukin 11 \\
\hline TXNIP & 2.8848 & 0.0006 & 0.0176 & $1.54 \mathrm{E}-05$ & Thioredoxin interacting protein \\
\hline AIM1L & 0.4801 & 0.0071 & 2.1508 & 0.0013 & Absent in melanoma 1-like \\
\hline KLK1 & 0.4736 & 0.0039 & 2.7208 & 0.0009 & Kallikrein 1 \\
\hline RAET1E & 0.4116 & 0.0027 & 6.1737 & 0.0017 & Retinoic acid early transcript $1 \mathrm{E}$ \\
\hline KCNQ2 & 0.3869 & 0.0009 & 2.6920 & 0.0001 & Potassium voltage-gated channel, KQT-like subfamily, member 2 \\
\hline NES & 0.3735 & 0.0015 & 2.9488 & 0.0001 & Nestin \\
\hline HSPA2 & 0.3183 & 0.0009 & 2.1676 & 0.0068 & Heat shock $70 \mathrm{kDa}$ protein 2 \\
\hline SUSD2 & 0.3074 & 0.0017 & 2.5593 & $3.79 \mathrm{E}-06$ & Sushi domain containing 2 \\
\hline MFAP5 & 0.3055 & 0.0049 & 3.1193 & 0.0043 & Microfibrillar associated protein 5 \\
\hline $\mathrm{OLAH}$ & 0.2559 & $8.55 \mathrm{E}-05$ & 2.3232 & 0.0032 & Oleoyl-ACP hydrolase \\
\hline ALPPL2 & 0.2195 & 0.0004 & 2.0811 & 0.0004 & Alkaline phosphatase, placental-like 2 \\
\hline
\end{tabular}

BGC-823/MTA2: $32.97 \pm 0.40$ vs. $34.93 \pm 1.15, \quad \mathrm{P}=0.049$, Figure 4B). IHC showed that IL-11 expression was reduced in SGC-7901/shMTA2 xenografts, and was significantly increased in BGC-823/MTA2 xenografts (Figure 4C).

\section{IL-11 expression was reduced by HDAC inhibitor SAHA} After SAHA treatment ( $4 \mu \mathrm{M}$ for $24 \mathrm{~h})$, IL-11 mRNA in SGC-7901/NC cells was significantly decreased $(P<$ 0.01 ), and similar with the level in SGC-7901/shMTA2 cells treated by DMSO. In BGC-823/NC cells, IL-11 mRNA was not changed by SAHA $(P>0.05)$, while its expression in BGC-823/MTA2/SAHA group was significantly decreased $(P<0.01$, Figure $4 \mathrm{D})$.

\section{IL-11 recovered colony formation capacity of MTA2 knockdown cells}

SGC-7901/shMTA2 and SGC-7901/NC cells were treated by rhIL-11 (100 ng/ml). In cell proliferation assay, cell growth rates were not significantly changed among cells treated by rhIL-11 or PBS (Figure 5A).

In colony formation assay, the colony number of SGC7901/shMTA2 cells treated by rhIL-11 was more than those treated by PBS (SGC-7901/shMTA2: rhIL-11 vs. PBS, $25.6 \pm 1.5$ vs. $20.0 \pm 2.3, P<0.01)$, and was similar with the number of SGC-7901/NC/PBS group (24.4 \pm $4.1, P=0.566)$. The number of colonies of SGC-7901/ NC cells treated by rhIL-11 was also more than those treated by PBS (SGC-7901/NC: rhIL-11 vs. PBS, $30.4 \pm$ 4.2 vs. $24.4 \pm 4.1, P<0.01$, Figure $5 \mathrm{C})$. The average size of colonies was also larger in SGC-7901/shMTA2/rhIL11 group than that in SGC-7901/shMTA2/PBS group (14.6 \pm 5.0 vs. $12.6 \pm 3.3, P=0.004$, Figure 5D).

\section{Discussion}

Herein, we found that MTA2 overexpression in gastric cancer increased colony formation in vitro and tumor growth in vivo, including either subcutaneous xenografts or pulmonary metastases. By genome expression analysis, IL-11 expression was found to be in accordance with down- and up-regulation of MTA2. Recombinant human IL-11 treatment could recover colony formation capacity of MTA2 knockdown cells.

The biological functions of MTA2 in cancer have not been fully investigated. MTA2 was first identified in quickly dividing cells in cervical cancer [9]. In nonsmall cell lung cancer, ki-67 index in MTA2 positive tissues was higher than that in negative ones [6]. MTA2 overexpression could increase colony formation of osteosarcoma cells SaoS-2 by inhibiting p53 activity, and also enhanced ER- $\alpha$ positive breast cancer cells growth $[12,13]$. On the other hand, in mouse breast cancer 4 T1 cells and glioma cells, it was reported that MTA2 knockdown impaired cell invasion and metastasis $[11,14]$. Those results showed that MTA2 played role in various malignant behaviors, including proliferation, invasion and metastasis. However, few studies assessed the biological function of MTA2 in both overexpression and knockdown manners.

We have previously reported that MTA2 knockdown in SGC-7901 and AGS gastric cancer cell lines with high MTA2 expression inhibited gastric cancer cell invasion and metastasis. Although attenuated cell colony formation and subcutaneous tumor growth were observed, these phenomena were attributed to the impaired cell invasion. Changes of cells morphology and reduced CD24 and MYLK expression further supported the conclusion that depression of cell mobility could be the 


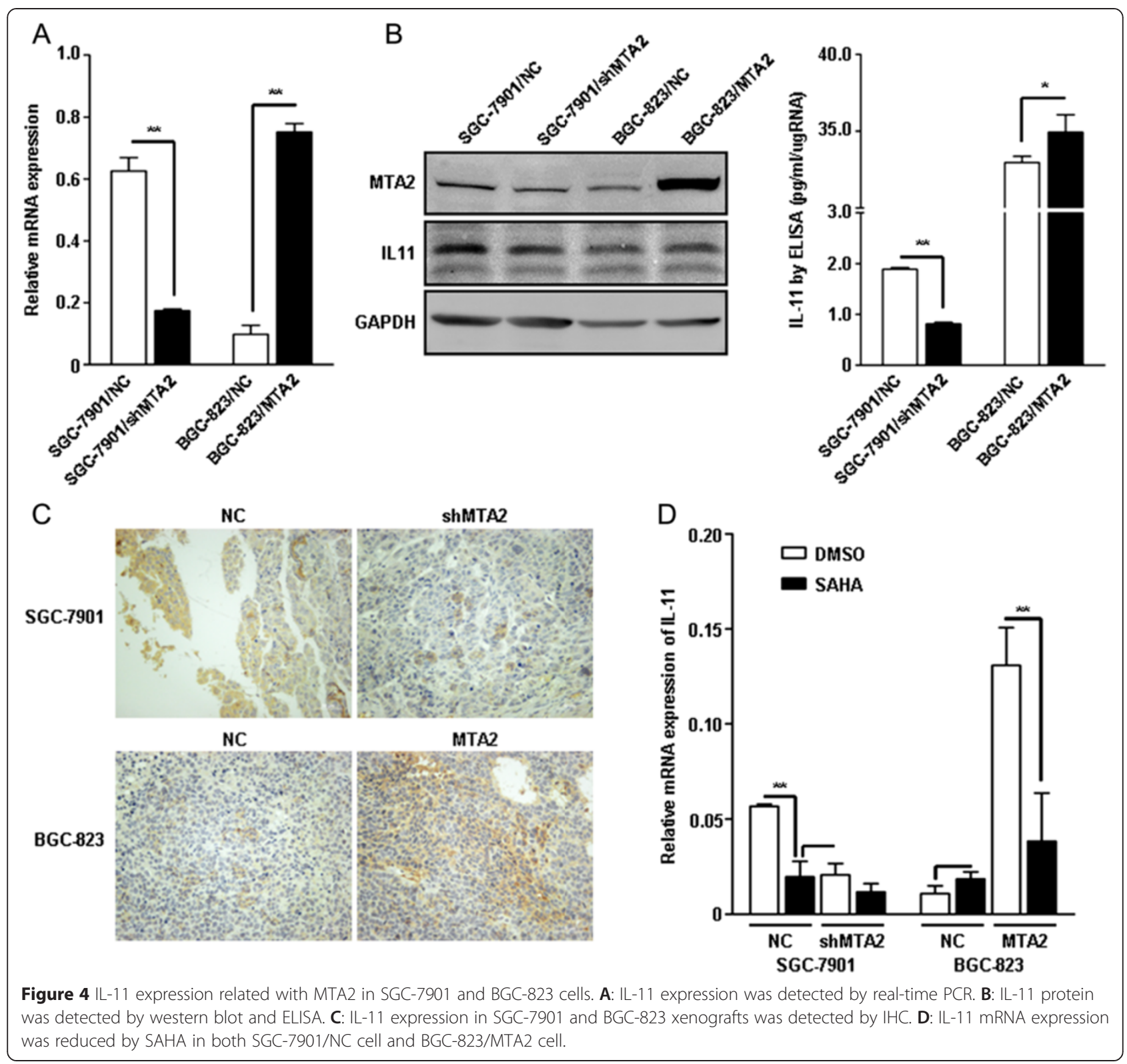

major consequence of MTA2 knockdown in gastric cancer cells [8].

To verify those phenomena, cell models based on gastric cancer cells with different genetic background were preferred to be used. Therefore, BGC-823 and MKN28 cell lines with low MTA2 expression was used to establish MTA2 knock-in cell models. From the results, we found that enhanced cell migration was not observed in MTA2 overexpression cells. Although CD24 and MYLK were identified by genome expression analysis in MTA2 knockdown cells as pervious reported, those two genes was not found in our MTA2 overexpression date set. Instead of cell invasion, colony formation in soft agar and tumor growth in vivo were significantly enhanced by
MTA2 overexpression, contrast to MTA2 knockdown. Positive correlation between Ki-67 and MTA2 was detected in both MTA2 knockdown and overexpression xenografts. Those results demonstrated that in gastric cancer cells, MTA2 was strongly related with cell colony formation and tumor growth. MTA2 participated in gastric cancer cell invasion, but might not be a dominant regulator.

The different results between cell proliferation assay and in vivo experiments indicated that interactions between tumor cells and its microenvironment could correlate with growth promoting effect of MTA2 [15]. Intercellular contact, growth factors, cytokines and extracellular matrix, all play important roles in tumor growth of gastric cancer [16]. In present study, IL-11 

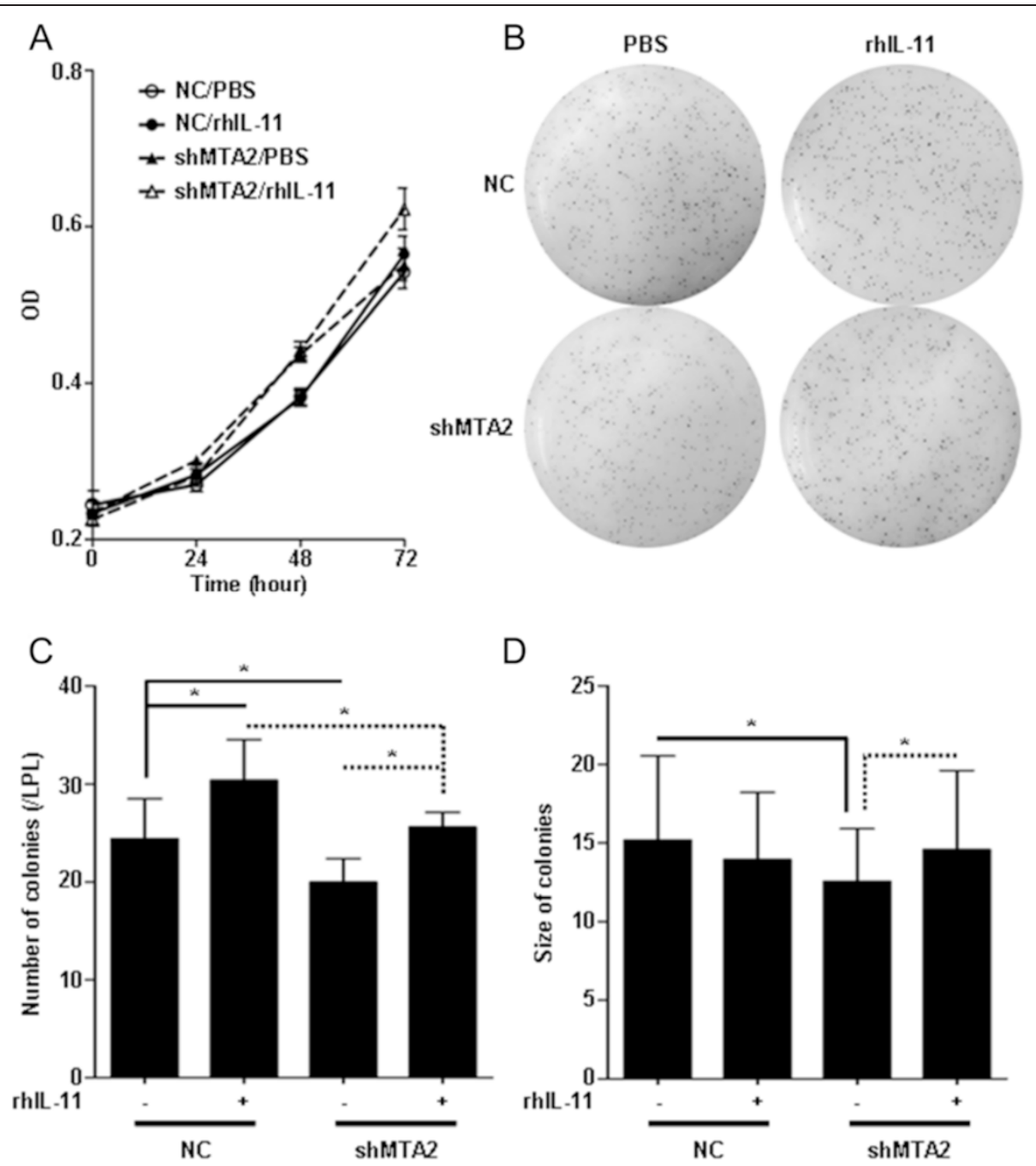

Figure 5 IL-11 recovered colony formation capacity of SGC-7901/shMTA2 cell. A: Growth curves of SGC-7901/NC and SGC-7901/shMTA2 cells were not affected by rhlL-11 treatment. B: Colony formation in soft agar was assessed after rhlL-11 treatment. C: Number of colonies in SGC-7901/shMTA2/L-11 group was more than it in SGC-7901/shMTA2/PBS group, and similar with SGC-7901/NC/PBS group. D: Size of colonies in SGC-7901/shMTA2/LL-11 group was larger than it in SGC-7901/shMTA2/PBS group.

expression was found related with MTA2 in gastric cancer cells by genome expression analysis, and was validated in both cell models and xenografts tissues.

IL-11 belongs to IL-6 cytokine family, which consisted of IL-6, IL-11, IL-27, IL-30, IL-31, oncostatin M, and others, and it can be secreted by cancer-associated fibroblasts, myeloid cells, and tumor cell itself $[17,18]$. Aberrant expression of IL-11 and its receptor IL-11R $\alpha$ was found in gastric cancer tissues, and correlated with Lauren's classification, tumor invasion and vessel infiltration [19]. In transgenic mice model carrying gp130 ${ }^{\mathrm{Y757F} / \mathrm{Y} 757 \mathrm{~F}}$, the IL-11 receptor with substitution of tyrosine (Y) 757 by phenylalanine (F), spontaneous gastric tumorigenesis is found. This mutation abolished negative feedback of gp130, resulting consistent activation of IL-11 downstream signaling pathway [20].
Gastric tumor formed in gp130 ${ }^{\mathrm{Y757F} / \mathrm{Y} 757 \mathrm{~F}}$ mice could be significantly abrogated by IL-11R $\alpha$ knock-out and also by IL11 signaling antagonist. Those results demonstrated that IL11 was one of the dominant factors in gastric cancer development and progression $[21,22]$.

To validate whether IL-11 was involved in cell colony formation regulated by MTA2, rhIL-11 was used to treat MTA2 knockdown cells in present study. Administration of rhIL-11 recovered colony formation ability of MTA2 knockdown cells and could further enhance it in NC cells, while cell proliferation was not effected by rhIL-11. Colony formation of BGC-823 cell could also enhanced by IL-11 treatment (Additional file 2: Figure S2). On the other hand, by using antibody to neutralize IL-11 function in BGC-823/ MTA2 cell, its colony formation was impaired (Additional 
file 3: Figure S3). The rescue assay suggested that MTA2 promoting gastric cancer cell colony formation might partially through IL-11 as a downstream effector.

The mechanisms of MTA2 in regulating gene expression are currently obscure. Because of its role in NuRD complex to maintain HDAC activity, the impact of MTA2 on IL-11 expression could partially via HDAC pathway. Therefore, we used HDAC inhibitor to simulate the status of MTA2 knockdown. After SAHA treatment, IL-11 expression was significantly reduced in SGC-7901/NC cell, and its level was similar to MTA2 knockdown cells. IL-11 expression in MTA2 overexpression cells was also decreased. Those results indicated that HDAC activity regulated by MTA2 might involve in regulating IL-11 expression.

Not only participates in NuRD complex formation, MTA2 could also form complexes with some transcription factors, resulting in gene transcriptional repression. Interaction of MTA2 and Twist participated in repressing Ecadherin promoter activity [11]. Binding with ER $\alpha$, MTA2 could repress its transcriptional activity in breast cancer cells [13]. In present study, increased expression of IL-11 in MTA2 overexpression cells indicated that MTA2 could promote specific gene expression, and its mechanism should be investigated in further studies.

Besides IL-11, several genes were identified by genome expression analysis. Some of those genes had been investigated in cancer cells, while the functions of others were still unclear. TXNIP is an endogenous antagonist of TRX, and can regulate cellular redox equilibrium by repressing TRX activity [23]. Expression of RAETI1E was correlated with poor prognosis of ovarian cancer patients [24]. Biological functions of HSPA2 had also been investigated in several tumors $[25,26]$. The roles of those genes in malignant behaviors regulated by MTA2 should be further explored.

\section{Conclusions}

MTA2 overexpression enhances colony formation and tumor growth of gastric cancer cells, but does not promote tumor migration and metastasis. IL-11 is one of the downstream effectors of MTA2 in regulating gastric cancer cells growth.

\section{Additional files}

Additional file 1: Figure S1. Immunofluorescence staining of MTA2 in MTA2 overexpression cells. Immunofluorescence staining showed that exogenous MTA2 localized in cellular nucleus.

Additional file 2: Figure S2. Colony formation of BGC-823 cells was enhanced by IL-11. BGC-823 cells in soft agar were treated by IL-11 and PBS, respectively. The method was described in manuscript. Results showed that number and size of colonies in BGC-823/LL-11 group was more than those in BGC-823/PBS group (IL-11 vs. PBS, number: $12.8 \pm 2.4$ vs. $9.2 \pm 1.3, P=0.018$; size $70.0 \pm 47.8$ vs. $50.0 \pm 34.8, P<0.001)$.

Additional file 3: Figure S3. Colony formation of BGC-823/MTA2 cells was reduced by IL-11 antibody. BGC-823/MTA2 cells in soft agar were treated by IL-11 antibody (R\&D systems, \#22626, $10 \mu \mathrm{g} / \mathrm{ml}$ ) and PBS, respectively. Results showed that number of colonies in BGC-823/MTA2/ anti-IL11 group was less than those in BGC-823/MTA2/PBS group (5.8 \pm 0.8 vs. $13.6 \pm 3.4, P=0.001)$. Size of colonies between those two groups was similar (30.4 \pm 19.4 vs. $36.2 \pm 28.9, P=0.194)$.

\section{Abbreviations}

NC: Negative control; MTA2: Metastasis associated 1 family, member 2; NuRD: Nucleosome remodeling and histone deacetylation; HDAC: Histone deacetylase.

\section{Competing interests}

The authors declare that they have no competing interests.

\section{Authors' contributions}

Conceived and designed the experiments: CZ, JZ, YY and ZZ. Performed the experiments: JJ, QC, MS and XC. Analyzed the data: CZ, JJ and JZ. Wrote the manuscript: CZ and JZ. All authors read and approved the final manuscript.

\section{Authors' information}

Chenfei Zhou and Jun Ji co-first authors.

\section{Acknowledgments}

The study was supported by National Science Foundation of China (81372645) and Shanghai Natural Science Foundation from municiple government (13ZR1425900) and Shanghai Jiao Tong University School of Medicine Science and Technology Foundation (13XJ10035) and FONG SHU FOOK TONG Foundation and National Key Clinical Discipline (Oncology) to J. Zhang. This study was also partially supported by the Chinese National High Tech Program (2012AA02A504, 2012AA02A203), the National Science

Foundation of China $(81172329,81372644)$ to Y. Yu.

\section{Author details}

${ }^{1}$ Department of Oncology, Ruijin Hospital, Shanghai Jiaotong University School of Medicine, No. 197 Ruijin er Road, Shanghai 200025, China. ${ }^{2}$ Department of Surgery, Shanghai Institute of Digestive Surgery, Ruijin Hospital, Shanghai Jiaotong University School of Medicine, No. 197 Ruijin er Road, Shanghai 200025, China.

Received: 17 December 2014 Accepted: 24 April 2015

Published online: 02 May 2015

\section{Reference}

1. Hartgrink HH, Jansen EP, van Grieken NC, van de Velde CJ. Gastric cancer. Lancet. 2009;374(9688):477-90.

2. Chen W, Zheng R, Zhang S, Zhao P, Zeng H, Zou X, et al. Annual report on status of cancer in China, 2010. Chin J Cancer Res. 2014;26(1):48-58.

3. Lordick F, Allum W, Carneiro F, Mitry E, Tabernero J, Tan P, et al. Unmet needs and challenges in gastric cancer: the way forward. Cancer Treat Rev. 2014;40(6):692-700.

4. Manavathi B, Kumar R. Metastasis tumor antigens, an emerging family of multifaceted master coregulators. J Biol Chem. 2007;282(3):1529-33.

5. Ji Y, Zhang P, Lu Y, Ma D. Expression of MTA2 gene in ovarian epithelial cancer and its clinical implication. J Huazhong Univ Sci Technolog Med Sci. 2006;26(3):359-62.

6. Liu SL, Han Y, Zhang Y, Xie CY, Wang EH, Miao Y, et al. Expression of metastasis-associated protein 2 (MTA2) might predict proliferation in non-small cell lung cancer. Target Oncol. 2012;7(2):135-43.

7. Chen DW, Fan YF, Li J, Jiang XX. MTA2 expression is a novel prognostic marker for pancreatic ductal adenocarcinoma. Tumour Biol. 2013;34(3):1553-7.

8. Zhou C, Ji J, Cai Q, Shi M, Chen X, Yu Y, et al. MTA2 promotes gastric cancer cells invasion and is transcriptionally regulated by Sp1. Mol Cancer. 2013;12(1):102.

9. Zhang $Y, \mathrm{Ng} \mathrm{HH}$, Erdjument-Bromage H, Tempst P, Bird A, Reinberg D. Analysis of the NuRD subunits reveals a histone deacetylase core complex and a connection with DNA methylation. Genes Dev. 1999;13(15):1924-35.

10. Bowen NJ, Fujita N, Kajita M, Wade PA. Mi-2/NuRD: multiple complexes for many purposes. Biochim Biophys Acta. 2004;1677(1-3):52-7.

11. Fu J, Qin L, He T, Qin J, Hong J, Wong J, et al. The TWIST/Mi2/NuRD protein complex and its essential role in cancer metastasis. Cell Res. 2011;21(2):275-89. 
12. Luo J, Su F, Chen D, Shiloh A, Gu W. Deacetylation of p53 modulates its effect on cell growth and apoptosis. Nature. 2000;408(6810):377-81.

13. Cui Y, Niu A, Pestell R, Kumar R, Curran EM, Liu Y, et al. Metastasis-associated protein 2 is a repressor of estrogen receptor alpha whose overexpression leads to estrogen-independent growth of human breast cancer cells. Mol Endocrinol. 2006;20(9):2020-35.

14. Cheng CY, Chou YE, Ko CP, Yang SF, Hsieh SC, Lin CL, et al. Metastasis tumor-associated protein-2 knockdown suppresses the proliferation and invasion of human glioma cells in vitro and in vivo. J Neurooncol. 2014;120(2):273-81.

15. Hanahan D, Weinberg RA. Hallmarks of cancer: the next generation Cell. 2011;144(5):646-74.

16. Ungefroren $\mathrm{H}$, Sebens $\mathrm{S}$, Seidl D, Lehnert $\mathrm{H}$, Hass R. Interaction of tumor cells with the microenvironment. Cell Commun Signal. 2011;9:18.

17. Putoczki T, Ernst M. More than a sidekick: the IL-6 family cytokine IL-11 links inflammation to cancer. J Leukoc Biol. 2010;88(6):1109-17.

18. Garbers C, Scheller J. Interleukin-6 and interleukin-11: same same but different. Biol Chem. 2013;394(9):1145-61.

19. Nakayama T, Yoshizaki A, Izumida S, Suehiro T, Miura S, Uemura T, et al. Expression of interleukin-11 (IL-11) and IL-11 receptor alpha in human gastric carcinoma and IL-11 upregulates the invasive activity of human gastric carcinoma cells. Int J Oncol. 2007;30(4):825-33.

20. Ernst M, Najdovska M, Grail D, Lundgren-May T, Buchert M, Tye $H$, et al. STAT3 and STAT1 mediate IL-11-dependent and inflammation-associated gastric tumorigenesis in gp130 receptor mutant mice. J Clin Invest. 2008;118(5):1727-38

21. Putoczki TL, Thiem S, Loving A, Busuttil RA, Wilson NJ, Ziegler PK, et al. Interleukin-11 is the dominant IL-6 family cytokine during gastrointestinal tumorigenesis and can be targeted therapeutically. Cancer Cell. 2013:24(2):257-71

22. Grivennikov SI. IL-11: a prominent pro-tumorigenic member of the IL-6 family. Cancer Cell. 2013;24(2):145-7.

23. Spindel ON, World C, Berk BC. Thioredoxin interacting protein: redox dependent and independent regulatory mechanisms. Antioxid Redox Signal. 2012;16(6):587-96.

24. McGilvray RW, Eagle RA, Rolland P, Jafferji I, Trowsdale J, Durrant LG. ULBP2 and RAET1E NKG2D ligands are independent predictors of poor prognosis in ovarian cancer patients. Int J Cancer. 2010;127(6):1412-20.

25. Scieglinska D, Gogler-Piglowska A, Butkiewicz D, Chekan M, Malusecka E, Harasim J, et al. HSPA2 is expressed in human tumors and correlates with clinical features in non-small cell lung carcinoma patients. Anticancer Res. 2014:34(6):2833-40

26. Scieglinska D, Piglowski W, Mazurek A, Malusecka E, Zebracka J, Filipczak P, et al. The HspA2 protein localizes in nucleoli and centrosomes of heat shocked cancer cells. J Cell Biochem. 2008;104(6):2193-206.

\section{Submit your next manuscript to BioMed Central and take full advantage of:}

- Convenient online submission

- Thorough peer review

- No space constraints or color figure charges

- Immediate publication on acceptance

- Inclusion in PubMed, CAS, Scopus and Google Scholar

- Research which is freely available for redistribution

Submit your manuscript at www.biomedcentral.com/submit 